\title{
Core-shell effects of functionalized oxide nanoparticles inside long-range meso-ordered spray-dried silica spheres
}

\author{
Niki Baccile $\cdot$ Anna Fischer $\cdot$ Beatriz Julián-López $\cdot$ \\ David Grosso · Clément Sanchez
}

Received: 27 February 2008/ Accepted: 28 April 2008/Published online: 17 May 2008

(C) The Author(s) 2008

\begin{abstract}
Core-shell and homogeneous distributions of functionalized cerium oxide nanoparticles within spraydried mesostructured silica spheres are achieved by modification of synthesis parameters such as the templating agent and nanoparticle capping functions.
\end{abstract}

Keywords Mesoporous silica - Embedded nanoparticles · Functionalized nanoparticles · Evaporation induced self assembly $\cdot$ Spray-drying

In the past 15 years a large number of studies has been focused on long-range ordered mesoporous materials obtained in solution through the self-assembly of a variety of amphiphilic templating agents with a growing inorganic phase [1]. Recently, several works showed that their well known structural characteristics (high surface area, tunable

Electronic supplementary material The online version of this article (doi:10.1007/s10971-008-1762-8) contains supplementary material, which is available to authorized users.

N. Baccile · A. Fischer · B. Julián-López · D. Grosso ·

C. Sanchez $(\square)$

LCMCP, UPMC-CNRS, 4, Place Jussieu, T54 E5 75252 Paris

Cedex 05, France

e-mail: clement.sanchez@upmc.fr

Present Address:

N. Baccile - A. Fischer

Max-Planck Institute Colloid Department, MPI Campus Golm,

14476 Golm, Germany

e-mail: niki.baccile@mpikg.mpg.de

Present Address:

B. Julián-López

Dpto. Química Inorgánica y Orgánica, Universitat Jaume I,

Avda. Sos Baynat s/n, 12071 Castellon, Spain geometry of the porous network, narrow pore size distribution) [2] are preserved and enhanced by mean of a spray drying process, which revealed to be interesting for its ease and possibility to obtain spherical particles. In Refs. [2c, 3], experimental conditions as well as different structuring agents were succesfully tested while in Ref. [4] SAXS, TEM and mainly solid state NMR were used to better understand the process of particle and mesophase formation with time. As far as the last point is concerned, Boissière et al. [5] used time-resolved in-situ SAXS to determine the required conditions for the mesophase to form. Regarding applications, spherical shaping and possibility to embed maghemite nanoparticles within a mesoporous silica matrix readily showed an interest in combined applications like drug delivery and magnetic resonance imaging [6] while catalysis was rather explored in presence of Pd nanoparticles [7] and nanowires [8]. In these cases, the way in which nanoparticles are distributed inside the silica particles is of primary importance according to the final application. For instance, in catalytic application, one could hope to enhance interactions between external fluids and embedded nanoparticles by placing them in the silica surface rather than in its bulk. In this way, diffusion paths are limited to the external crust. On the contrary, if the application involves magnetic or optical properties, an homogenous distribution within the silica bulk is required in order to avoid destructive interactions (magnetic coupling, optical quenching...) origined from clustering of nanoparticles.

For these reasons, we want to show the possibility of tuning the spatial distribution of plain and functionalized cerium oxide nanoparticles inside spherical mesostructured silica particles produced by the spray-driyng technique. The goal is double: firstly we would like to show some tricky interactions effects between functionalized 
nanoparticles and surfactants employed to structure silica; secondly, we would like to open some perspectives as far as possibilities of easy controlling the spatial distribution of embedded nanoparticles.

Surface modification of cerium oxide, $\mathrm{CeO}_{2}{ }^{1}$, nanoparticles (used as received) was accomplished according to the procedure developped in Ref. [9]. Calculated ceria mass with respect to TEOS is about $10 \%$. Surface modification is performed by addition of phenyl phosphonic acid (PPA) and 3-amino propyl phosphonic acid. When PPA is used, homogenous dispersion of nanoparticles can only be obtained by addition of THF while no THF is employed with amino-propyl phosphonic acid. Ceria solution is then mixed with a CTAB (cetyltrimethylammonium bromideAldrich)/water/HCl/ethanol solution to which TEOS (tetraethoxysilane-Aldrich) is finally added. Final masses (in grams) of the spray-dried sol are the following:

\begin{tabular}{llll}
\hline $\mathrm{CeO}_{2}$ & PPA & THF & TEOS \\
0.132 & 0.012 & 0.338 & 4.690 \\
$\mathrm{CTAB}$ & $\mathrm{EtOH}$ & $\mathrm{H}_{2} \mathrm{O}$ & $\mathrm{HCl}(\mu \mathrm{L})$ \\
1.480 & 30.460 & 15.890 & 36 \\
\hline
\end{tabular}

Functionalization was checked by FT-IR spectroscopy for both phenyl and amino modified systems, as shown, respectively, in Figs. S1 and S2 in electronic supplementary information (ESI). Disappearing of FTIR bands of free $\mathrm{P}-\mathrm{O}$ vibration at $2,300 \mathrm{~cm}^{-1}$ region and lack of free $\mathrm{P}=\mathrm{O}$ band in the $1,200 \mathrm{~cm}^{-1}$ region suggest a tridentate binding of phosphonate group. For the amino modification (Fig. S2, ESI), additional peaks at 1,047 and $1,090 \mathrm{~cm}^{-1}$ suggest the presence of asymetric stretching vibrations of $\mathrm{P}-\mathrm{O}$ as detected in $\mathrm{PO}_{3}^{2-}$ group, though $\mathrm{C}-\mathrm{N}$ vibrations also resonate in this region. Unfortunately, these same vibrations are masked by the strong peak at $1,070 \mathrm{~cm}^{-1}$ and belonging to THF.

FT-IR gives a primary evidence of functionalization but exact mechanism and quantification is far from being determined, being this a very hard task to put in evidence [10]. Solution of functionalized cerium oxide nanoparticles is finally added to precursor sol mixture and eventually set in the spraying chamber. Experimental apparatus was described elsewhere [2c]; here, we have set the drying chamber temperature to $150{ }^{\circ} \mathrm{C}$, the furnace temperature to $350{ }^{\circ} \mathrm{C}$ while the relative humidity was set to $20 \%$. X-ray diffraction (XRD) was performed in a $\theta-2 \theta$ geometry $(\mathrm{Cu}$ $\mathrm{K} \alpha, \lambda \sim 0.154 \mathrm{~nm}$ ) (Philips PW 1820) while Transmission

\footnotetext{
${ }^{1} \mathrm{CeO}_{2}$ nanoparticles are obtained from the hydrate $\mathrm{CeO}_{2}(\mathrm{H}-$ $\left.\mathrm{NO}_{3}\right) 0.5\left(\mathrm{H}_{2} \mathrm{O}\right)_{4}$ delivered by Rhodia.
}

Electron Microscopy (TEM) was done on a Philips CM20 $(200 \mathrm{kV})$ microscope coupled with a spectrometer for Energy Dispersion of X-ray (EDX) analysis (CME, Université d'Orléans, France). Nitrogen adsorption/desorption isotherms were realized on a Micromeritics ASAP 2100 machine.

Figure 1 shows the $\mathrm{X}$-ray diffraction patterns of assynthesized mesostructured silica materials and in presence of amino-modified and phenyl-modified cerium oxide particles. All patterns show three low-angle diffraction peaks which can be safely indexed as the (10), (11) and (20) reflections of a long-range ordered 2D-hexagonal mesophase, that is a hexagonal close packing of micellar cylinders, in complete agreement with previous observation [2c]. Addition of the nanoparticles does not modify the long-range order within the material, both when hydrophilic and hydrophobic functions are used, but slight decrease of hexagonal cell parameter from 47 to $40 \AA$ is observed when ceria is functionalized with amino and phenyl group.

A possibility exists that nanoparticles with hydrophobic functions are embedded within micelles but this is excluded by the nitrogen adsorption/desorption isotherms shown in Fig. 2, where adsorbed volumes are of the order of $450 \mathrm{~cm}^{3} / \mathrm{g}$ in both calcined samples and BET surface areas range about $1,100 \mathrm{~m}^{2} / \mathrm{g}$. The capillary condensation occurs at values below $\mathrm{P} / \mathrm{P}_{0}=0.4$, as typically observed for CTAB-templated materials. In addition, no real difference in the isotherms is observed meaning that the presence of nanoparticles does not interfere with mesostructuring process.

Electron microscopy images of all materials are shown in Fig. 3. In all cases one remarks both the dispersion of

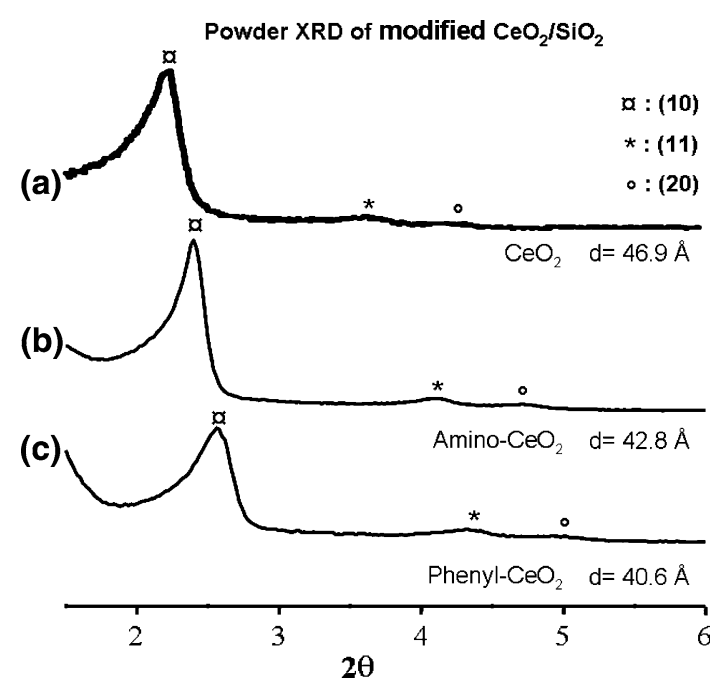

Fig. 1 X-ray diffraction patterns of spray-dried mesostructured silica particles embedding cerium oxide nanoparticles with (a) no functionalization and (b) amino and (c) phenyl functionalizations 

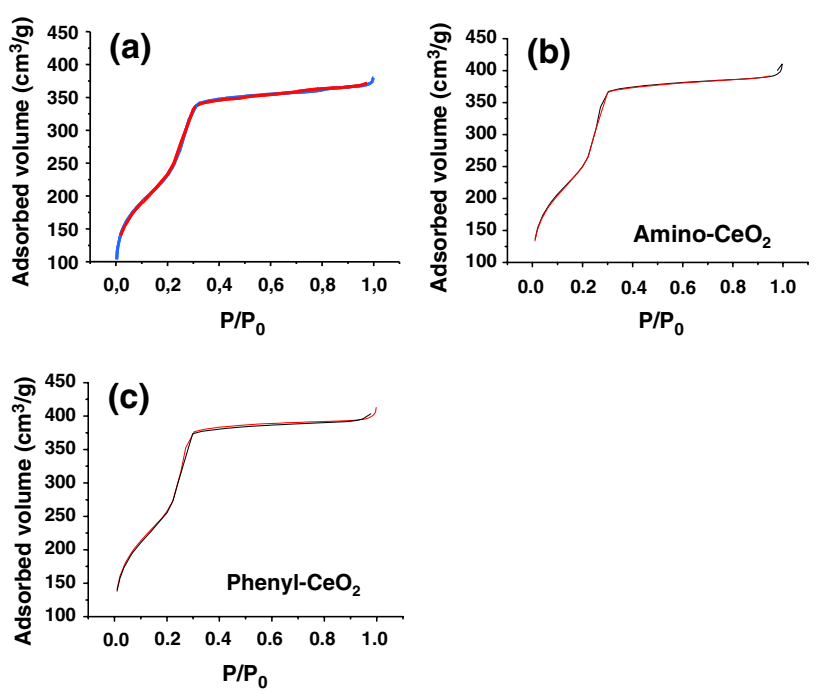

Fig. $2 \mathrm{~N}_{2}$ adsorption/desorption isotherms of spray-dried mesoporous silica particles embedding (a) non-functionalized and (b) aminoand (c) phenyl-functionalized cerium oxide nanoparticles

ceria nanoparticles inside the silica matrix as well as the good quality of the long-range order of CTAB micelles. Embedding of cerium oxide was verified by the presence of
CeL peak obtained by EDX measurements shown in Fig. 3i and taken during TEM measurements, as shown by the presence of the $\mathrm{CuK}$ belonging to the copper grid support used for measuring.

When no modification is performed $(a, b)$, ceria nanoparticles are quite well dispersed throughout the mesostructured silica matrix. No preferential site seems to be preferred by nanoceria to settle during the mesostructuring process. When the phenyl group is used as surface modifier, cerium oxide aggregates are also homogeously dispersed within the silica matrix, as Fig. 3c, d shows. Dispersion is very similar to system without modification. When zooming out on Fig. 3e, an estimation of nanoceria dimension hands out values between 5 and $8 \mathrm{~nm}$, which makes these objects too large to fit within CTAB templated pores, whose average pore diameter from $\mathrm{N}_{2}$ adsorption/ desorption data is $2.3 \mathrm{~nm}$. Reasons of quite homogenous dispersion could be probably found in the interactions between silica and functionalized nanoaggregates. An interesting discussion on silica surface chemistry on similar materials is lead by Alonso et al. [4a], they observe a high percentage of unreacted ethoxy groups when initial sols is not aged enough time, as it occurs in our case. As far as
Fig. 3 Microtomed TEM images of mesostructured silica embedding: (a, b) nonmodified, $(\mathbf{c}-\mathbf{e})$ phenyl-modified and (f-h) amino-modified cerium oxide nanoparticles. (i) EDX spectrum of ceriacontaining silica obtained during TEM measurements
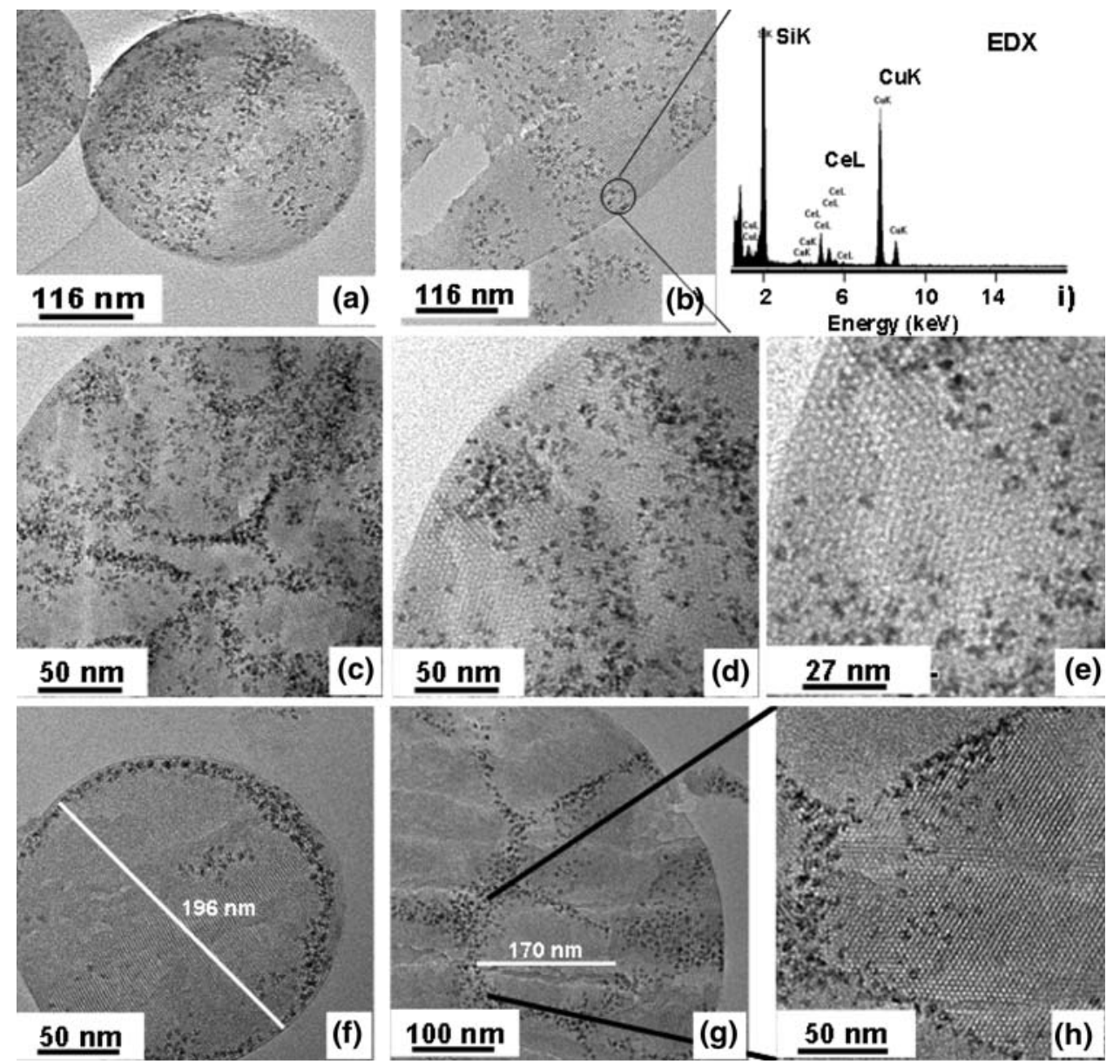
hydroxy groups are concerned, they attributed the low $\mathrm{OH}$ NMR signal to both water and silanols. It is known that hydrophilicity of silica surface in mesostructured powders vary very much between very acidic and very basic conditions [11]. Here, due to the mild initial $\mathrm{pH}$ and to the important presence of ethanol, it would be difficult to extrapolate a clear picture. Nevertheless, it would not be surprising that low percentages of silanols and adsorbed water combined to the existence of unreacted ethoxy groups may render an overall hydrophobicity of the material core. In addition, the homogenously dispersed and generalized presence of CTAB could definitely contribute to exclude possible hydrophilic areas and enhance hydrophobic interaction with phenyl-functionalized cerium particles.

Figure $3 f$ shows the amino modified nanoaggregates embedded in mesostructured silica particle of about $190 \mathrm{~nm}$ in diameter. Here, nanoceria mainly settle at the external silica/air interface. Such a radial distribution is also kept in larger silica spheres whose diameter is around $500 \mathrm{~nm}$. In Fig. 3g, h, meso-ordered domains surrounded by nanoceria form the core of the main silica particle indicating that coagulation among primary silica spheres whose diameter ranges between 170 and $200 \mathrm{~nm}$ during spray-drying occurred [12]. This observation, though trivial, shows that nanoceria actually migrates to the silica/air interface before silica condensation takes place and not after, since coagulation between droplets occurs in the first stages of the spraying process. More precisely, then, we should consider the migration of nanoceria taking place from the inner core of the liquid droplet towards the liquid/ air rather than a solid/air boundary.

Which parameters drive the migration of amino-functionalized ceria nanoparticles towards the outer droplet boundary? The localization of nanoparticles at the air/ droplet interface has already been described [13]. In their case, solvent evaporation rate was set to be the main parameter influencing the migration and aggregation of nanoparticles in a corona fashion. Parameters playing a crucial role seem to be the viscosity of the medium, evaporation rate of solvent and time needed to pass from liquid droplet to solid particle. In our system, all experimental conditions remain unchanged between nonfunctionalized and functionalized ceria embedding. The difference in the distribution behavior should be related to the departure of ethanol during drying and presence of the amino function. Since the synthesis occurs at low $\mathrm{pH}$, it is very likely that grafted amino groups are positively charged making the surface of nanoparticles virtually similar to the surfactant polar heads. Binks [14] has recently shown that nanoparticles and surfactants may behave similarly as far as segregation at interfaces is concerned but self-assembly only occurs for real amphiphiles. In our system, the high quantity of ethanol stabilizes CTAB molecules and ammonium-grafted nanoparticles before spraying. When ethanol evaporates, selfassembly into micelles occurs for CTAB molecules but not for functionalized nanoparticles, which segregate at the liquid/gas interface. The driving force for the particle migration can be found in the repulsion that may take place between positively charged amino functions and headgroups belonging to CTAB surfactant micelles. Verification of such an argument was tested by using neutral surfactants (results not shown), Brij58 and Pluronic P123 under the same synthesis conditions employed for CTAB. In any case a structured silica powder is obtained but surface modification did not influence at all the spatial distribution of ceria particles, which were always homogenously distributed inside silica particles.

In conclusion, we show that spatial distribution of functionalized nanoparticles within mesostructured CTABtemplated silica spheres obtained by spray drying can be varied by adjusting the type of surface function. When ceria nanoparticles are covered with hydrophobic phenylphosphonic acid or when they are used as-such, homogenous distribution inside mesostructured silica is obtained. On the contrary, when an amino-containing function is introduced at low $\mathrm{pH}$, migration towards the air/ droplet interface and a final core-shell type of the nanoparticles is eventually obtained.

Acknowledgements B. Julián thanks the financial support of the "Ramon y Cajal" Program (Spanish government) and the Bancaixa Foundation-Universitat Jaume I (P1 1B2007-47) project. N. Baccile thanks Prof. P. Innocenzi (LSMN, University of Sassari, Italy) and the EU Erasmus project for intra-European mobility.

Open Access This article is distributed under the terms of the Creative Commons Attribution Noncommercial License which permits any noncommercial use, distribution, and reproduction in any medium, provided the original author(s) and source are credited.

\section{References}

1. (a) Schuth F (2001) Stud Surf Sci Catal 135:1; (b) Tanev P, Butruille J-R, Pinnavaia TJ (1998) Chemistry of advanced materials: an overview. Wiley-VCH

2. (a) Lu Y, Fan H, Stump A, Ward TL, Rieker T, Brinker CJ (1999) Nature 398:223; (b) Brinker CJ, Lu Y, Sellinger A, Fan H (1999) Adv Mater 11:579; (c) Baccile N, Grosso D, Sanchez C (2003) J Mater Chem 13:3011

3. Andersson N, Alberius PCA, Pedersen JS, Bergström L (2004) Microporous Mesoporous Mater 72:175

4. (a) Alonso B, Douy A, Véron E, Perez J, Rager M-N, Massiot D (2004) J Mater Chem 14:2006-2016; (b) Alonso B, Véron E, Durand D, Massiot D, Clinard C (2007) Microporous Mesoporous Mater 106:76

5. Boissière C, Grosso D, Amenitsch H, Gibaud A, Coupé A, Baccile N, Sanchez C (2003) Chem Commun 2798 
6. Julián-López B, Boissiere C, Chaneac C, Grosso D, Vasseur S, Miraux S, Duguet E, Sanchez C (2007) J Mater Chem 17:15631569

7. Hampsey JE, Arsenault S, Hu Q, Lu Y (2005) Chem Mater $17: 2475-2480$

8. Borel MT, Ward TL, Fukuoka A, Datye AK (2004) Catal Lett 98:167-172

9. Bouchara A, Soler-Illia GJ de AA, Chane-Ching J-Y, Sanchez C (2002) Chem Commun 1234

10. van Lokeren L, Maheut G, Ribot F, Escax V, Verbruggen I, Sanchez C, Martins JC, Biesemans M, Willem R (2007) Chem Eur J 13:6957
11. Baccile N, Laurent G, Bonhomme C, Innocenzi P, Babonneau F (2007) Chem Mater 19:1343-1354

12. Kodas TT, Hampden-Smith MJ (1999) Aerosol processing of materials. Wiley-VCH

13. Sen D, Spalla O, Taché O, Haltebourg P, Thill A (2007) Langmuir 23:4296

14. Binks BP (2002) Curr Opin Colloid Interface Sci 7:21 